\title{
Effectiveness of Integrated Meta-Diagnostic Therapy on Cognitive Emotion Regulation and Psychological Resilience of Veterans' Spouses
}

\section{ART I C L E INF O}

\section{Article Type}

Original Research

\section{Authors}

Mozaffari V. ${ }^{1} M S c$

Ghorban Shiiroudi Sh. ${ }^{* 2} P h D$,

Shafiabadi A. ${ }^{3} \mathrm{PhD}$

How to cite this article
Mozaffari V, Ghorban Shiiroudi Sh,
Shafiabadi A. Effectiveness of Integ-
rated Meta-Diagnostic Therapy
on Cognitive Emotion Regulation
and Psychological Resilience of
Veterans' Spouses. Iranian Jo-
urnal of War \& Public Health.
$2020 ; 12(3): 149-155$.

${ }^{1}$ North Tehran Branch, Islamic Azad University, Tehran, Iran

${ }^{2}$ Department of Psychology, Tonekabon Branch, Islamic Azad University, Mazandaran, Iran

${ }^{3}$ Faculty of Humanities, Allameh Tabatabai University, Tehran, Iran

\section{*Correspondence}

Address: Islamic Azad University, Tonekabon Branch, Mazandaran, Iran. Postal Code: 4684161167. Phone: +98 (912) 1581822 Fax: -

drshohrehshiiroudi@gmail.com

\section{Article History}

Received: December 02, 2020

Accepted: January 23, 2021

ePublished: March 07, 2021

\section{A B S T R A C T}

Aims The spouses of psychiatric veterans are exposed to various psychological traumas due to their spouses' special circumstances. This study aimed to investigate the effectiveness of integrated meta-diagnostic treatment on veterans' spouses' cognitive emotion regulation and psychological resilience.

Materials \& Methods The present quasi-experimental study was conducted with a pretestposttest with a control group on the spouses of psychiatric veterans in Tehran. Using the voluntary sampling method from June to September 2016, by referring to the Tehran's District 4 Veterans Medical Centers and distributing voluntary forms among the veterans' spouses, 30 veteran spouses were selected from among the volunteers and randomly selected in experimental groups, and Controls were assigned. Cognitive emotion regulation questionnaire and resilience scale were used to collect data. The experimental group received integrated meta-diagnostic treatment for two and a half months in 10 90-minute sessions, one session per week. Data were analyzed using SPSS 23 statistical software and analysis of covariance.

Findings The mean scores of cognitive regulation of emotion and its dimensions and psychological resilience of veterans' spouses increased in the experimental group. 57\% changes in cognitive emotion regulation component $(\mathrm{F}=31.62 ; \mathrm{p}=0.0001), 52 \%$ changes in psychological resilience component $(F=26.05 ; p=0.0001), 37 \%$ changes in emotional inhibition dimension ( $F=13.21 ; \mathrm{p}=0.001)$ and $49 \%$ changes in the cognitive reassessment dimension $(\mathrm{F}=21.45 ; \mathrm{p}=0.0001)$ of veterans' spouses were explained by implementing integrated meta-diagnostic treatment.

Conclusion Integrated meta-diagnostic therapy can be used as an effective treatment to improve veterans' spouses' cognitive emotion regulation and psvchological resilience. Keywords Psychological Resilience; Unified Protocol; Emotion Regulation; Veteran's Wives

\section{I T A T I O N L I N K S}

[1] Effectiveness of cognitive mindfulness-based ... [2] The impact of life skills training ... [3] Effectiveness of acceptance and commitment ... [4] The comparison of the effectiveness ... [5] Prediction of emotional empathy in the wives ... [6] Examining emotion regulation in depression ... [7] Intergenerational transmission of emotion ... [8] Stress and delay discounting: The ... [9] Coping with the invisible enemy: The role of emotion ... [10] Emotion introspection and ... [11] Emotion regulation as a ... [12] Relationship between religious beliefs and ... [13] Well-being for Public ... [14] Promoting resilience among nursing ... [15] The moderating role of resiliency on the ... [16] Resilience processes in development ... [17] Effects of resilience training on the reduction ... [18] Sources of perceived social support on ... [19] Personality factors, student resiliency ... [20] Emotional disorders: A unified ... [21] Unified protocol for transdiagnostic ... [22] A systematic review of Unified Protocol ... [23] The effect of unfiled transdiagnostic ... [24] Effectiveness of transdiagnostic educational ... [25] The effectiveness of transdiagnostic treatment ... [26] Integrate metacognition for cognitive ... [27] The effectiveness of transdiagnostic ... [28] The effect of unified transdiagnostic ... [29] Treating depressive disorders with ... [30] Using the unified protocol for ... [31] A systematic review and meta-analysis of ... [32] Efficacy of the unified protocol for transdiagnostic ... [33] Secondary depression in transdiagnostic group ... [34] The utilization of unified protocols in ... [35] Finite-time and fixed-time bipartite ... [36] Analyzing Incomplete Political ... [37] Individual differences in two emotion ... [38] Relationship between emotion regulation and ... [39] Effectiveness of acceptance and commitment ... [40] Development of a new resilience scale ... [41] The effectiveness of spirituality therapy on psychological ... 
طولانىمدت در رنج هستند. در جنين وضعيتى كنشهاى روانى-

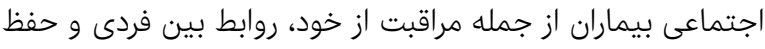

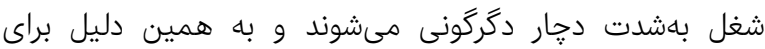

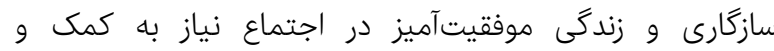

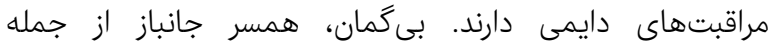

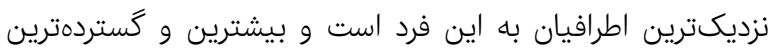

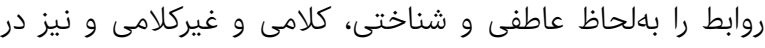

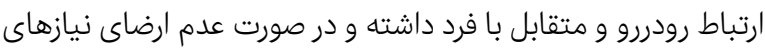

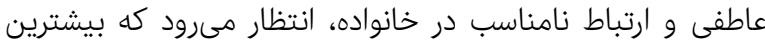

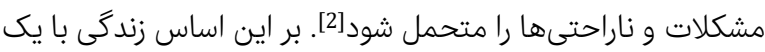

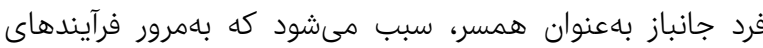

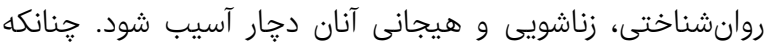

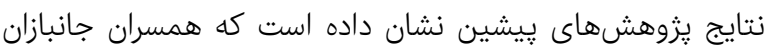

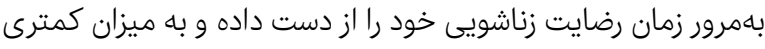

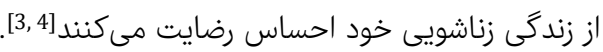

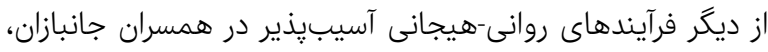

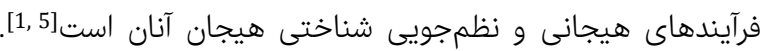

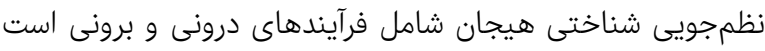

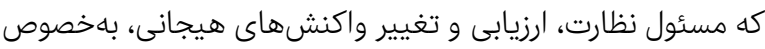

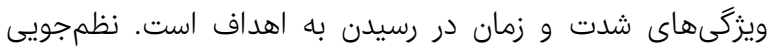

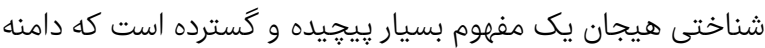

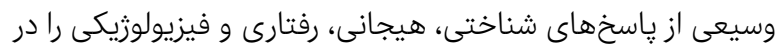
برمى

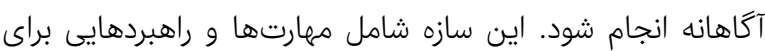
نظارت، ارزشيابى و تغيير واكنشهاى هيجانى است. راهبردهاى إنىاي

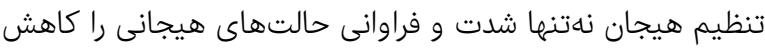

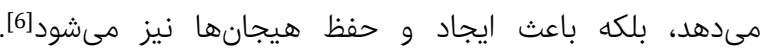

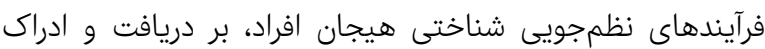

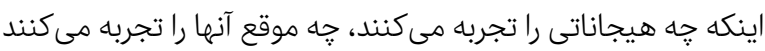

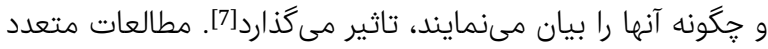

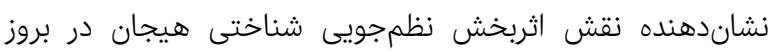

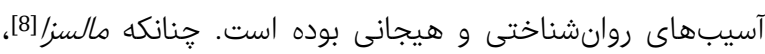

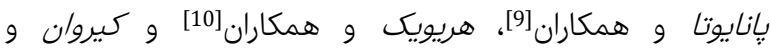

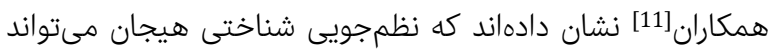

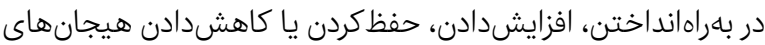

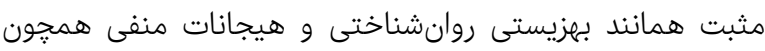

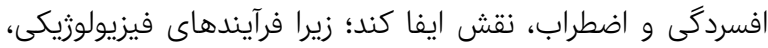

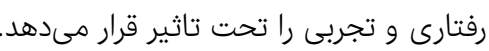

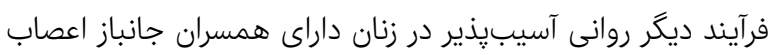

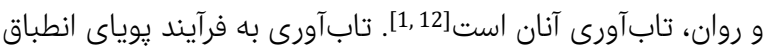

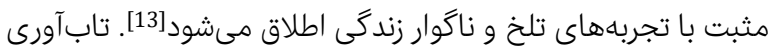

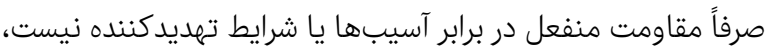

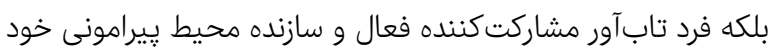

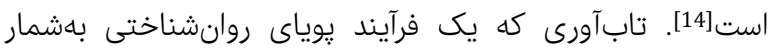

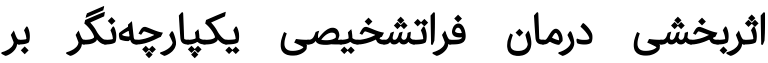

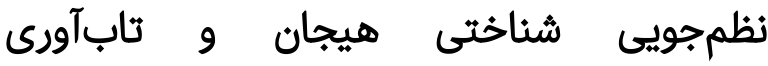 روانشناختى همسران جانبازان}

\author{
MSc واليه مظفرى \\ كروه مشاوره، واحد تهران شمال، دانشكًاه آزاد اسلامى،تهران، ايران
}

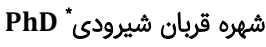

كروه روانشناسى، واحد تنكابن، دانشكاه آزاد اسلامى، مازندران، ايران

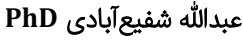

كروه مشاوره، دانشكده علوم انسانى، دانشَاه علامه طباطبايى، تهران، ايران

هكيده

اهداف: همسران جانبازان اعصاب و روان به دليل شرايط خاص همسران خود در

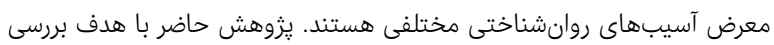

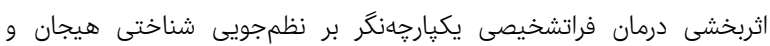

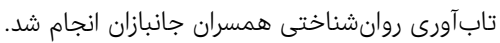

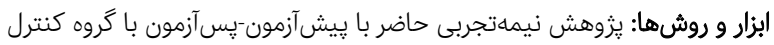

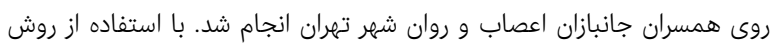

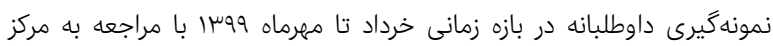

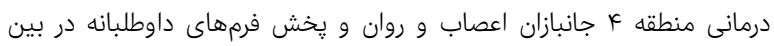

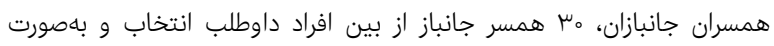

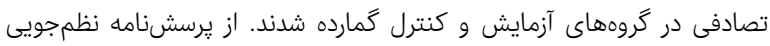

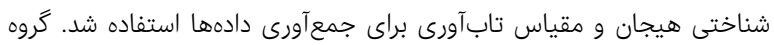

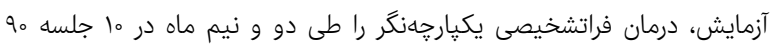

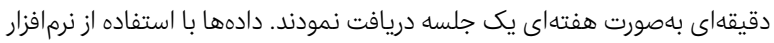

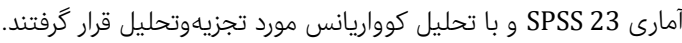

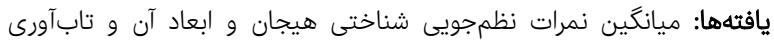

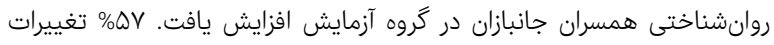

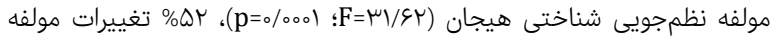

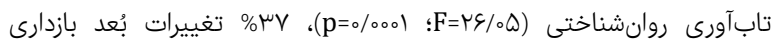

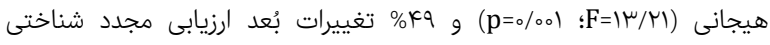

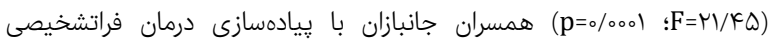

يكيارجهنكَ تبيين شد.

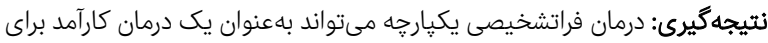

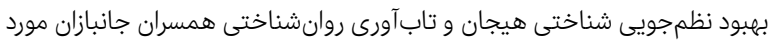

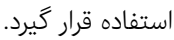

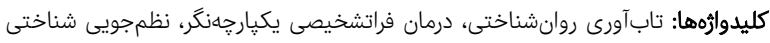

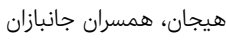

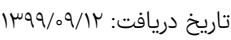

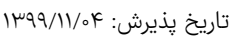
"نويسنده مسئول: لتايخ: drshohrehshiiroudi@gmail.com

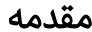

در ايران وجود هشت سال جنگ و استرسهاى ناشى از آن موجب

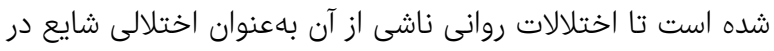

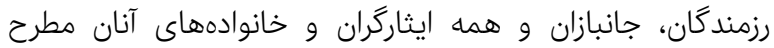

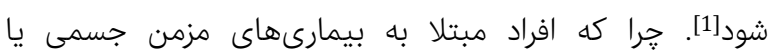
روانشناختى از اختلالات رفتارى، شناختى و روانشناختى شديد و 


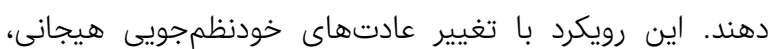
فراوانى و شدت استفاده از عادتهاى هيجانى ناسازكارانه را كاهش

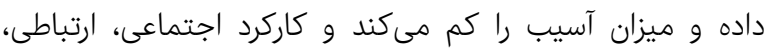

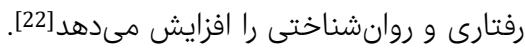

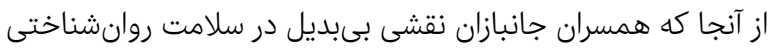

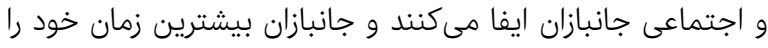

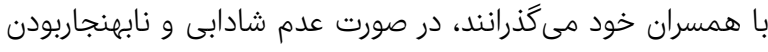

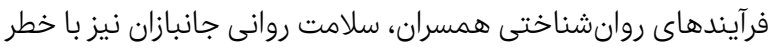

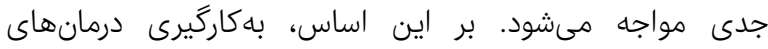

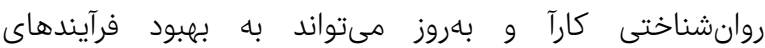

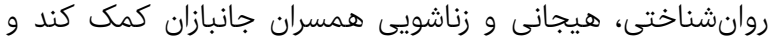

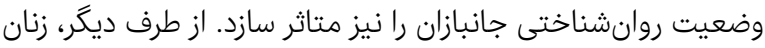

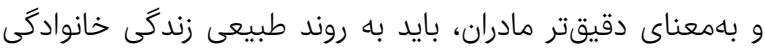

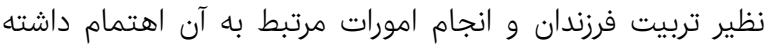

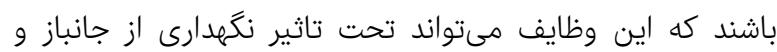

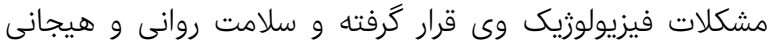

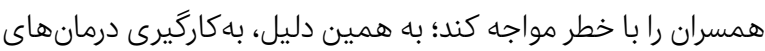
روانشناختى مناسب براى مادرانى كه شوهد جانباز دارند از اهميتى دانى دانى

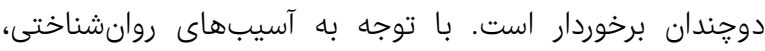

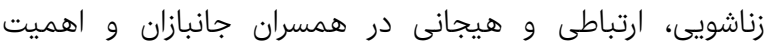

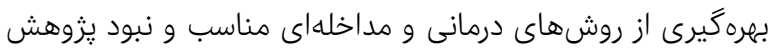

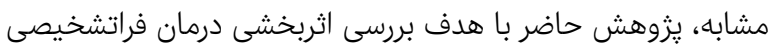

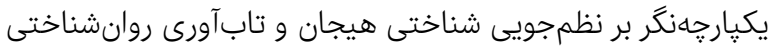

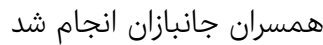

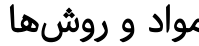

يزَوهش نيمهتجربى حاضر با پِيشآزمون-يسآزمون با كروه كنترل

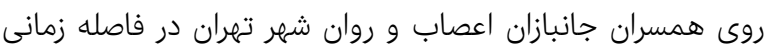

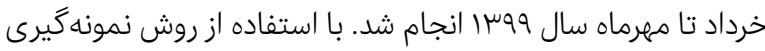

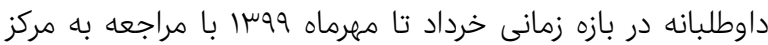

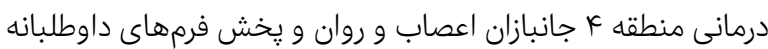

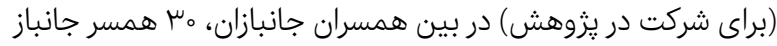

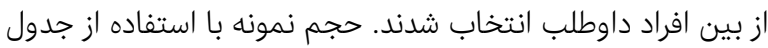

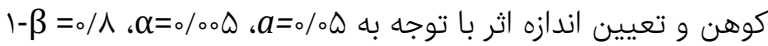

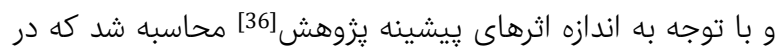

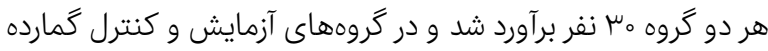

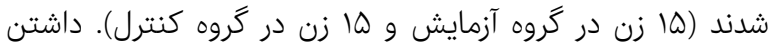

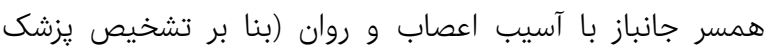

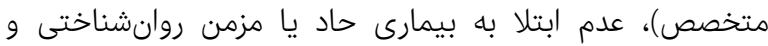

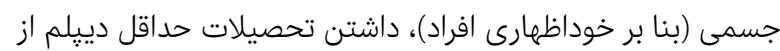

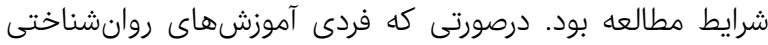

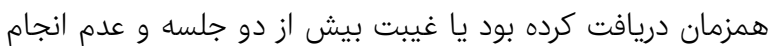
تكاليف محوله داشت، از تحقيق كنار كذاشته شد.
مىرود، در موقعيتهاى مختلف زندكى بهويزه مواقع بحرانى،

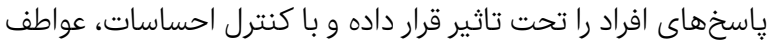

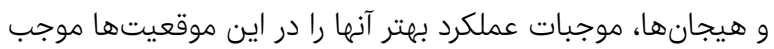

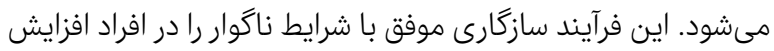

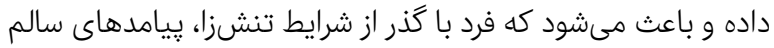

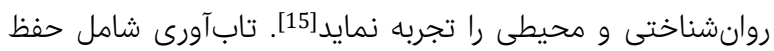

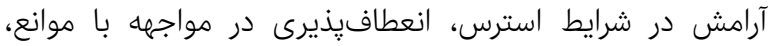

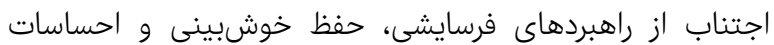

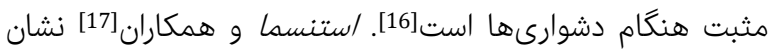
دادند كه تابآورى با استفاده از راهبردهاى كنارآمدن موثر مانند: راهبردهاى فعال، جستوجوى حمايت اجتماعى، خوددلكرمسازى و واني

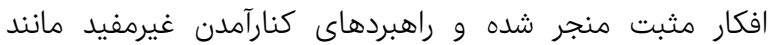

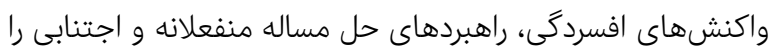

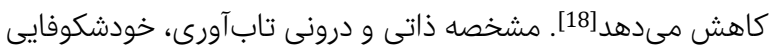

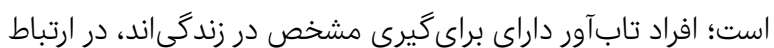

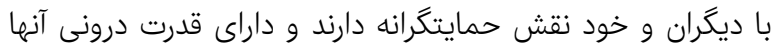

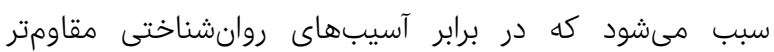

باشند]19]

روشهاى درمانى و آموزشى مختلفى براى حل مشكلات

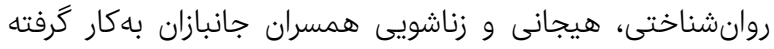

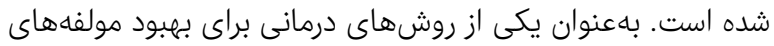

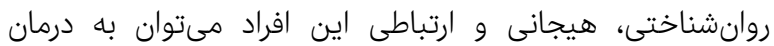

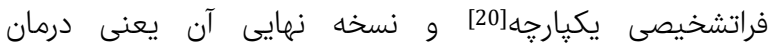

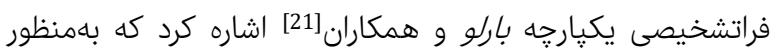

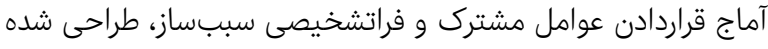

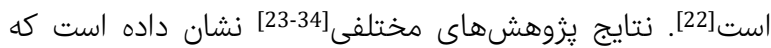

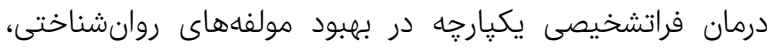

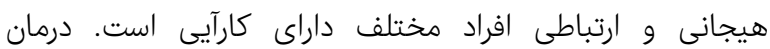

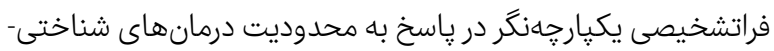

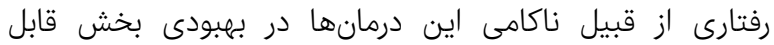

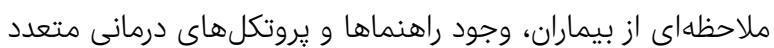

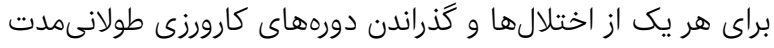
و متعدد براى هر يك از اختلالها و ايجاد سراد سردرًمى در در انتخاب

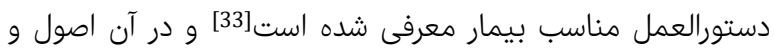

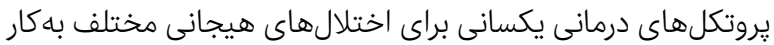

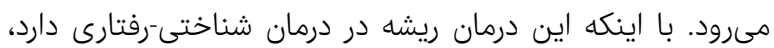

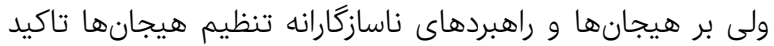

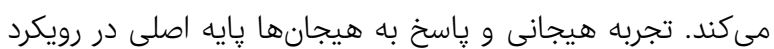

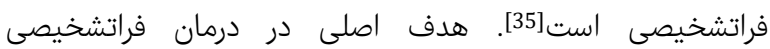
يكيارجهنكر اين است كه مراجعان مهارتهايى را كسب كنئن كهان

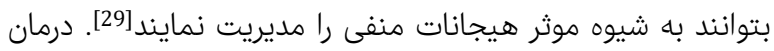

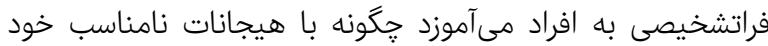

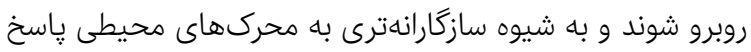




\begin{tabular}{|c|c|}
\hline \multicolumn{2}{|c|}{ جدول () خلاصه جلسات درمان فراتشخيصى يكيارجه [21] } \\
\hline شرح جلسه & جلسه هدف \\
\hline طول درمان، ارايه منطق درمان مشاركت و و دركيين اهدى بيماران درمان & اول افزايش انكيزه \\
\hline آموزش مدلى سيجانهانها و رديابى تجارب هيجانى هيجان و مدل و ARC. & دوم روانى آموزش \\
\hline 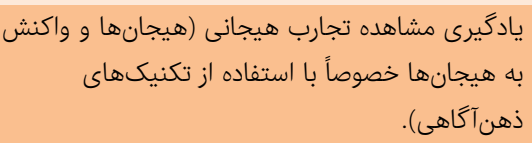 & سوم هيجانى آموز آهاهى \\
\hline 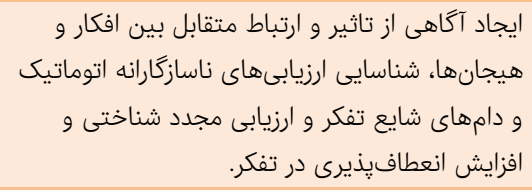 & جهارم ارزيابى مجدي و \\
\hline 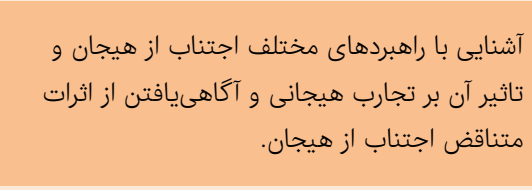 & شناسمايى الخوهاى \\
\hline 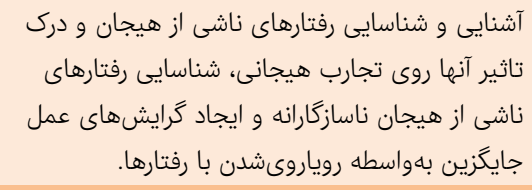 & شرم رفتارهاى ناشى \\
\hline 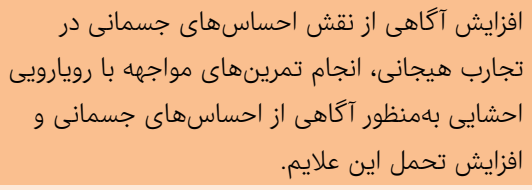 & 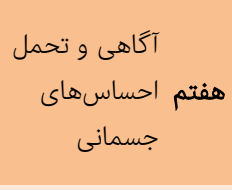 \\
\hline 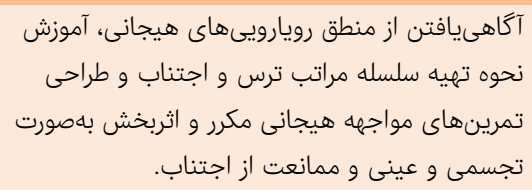 & و نهم هرم ريجان مبتنى بارويى \\
\hline مريشرورتهاى درمانى مفاهيم درمان و بحث در مورد بهبودى و & دهم عرد \\
\hline
\end{tabular}

از آزمون شإييرو-ويلك براى بررسى نرمالبودن توزيع دادهها، آزمون

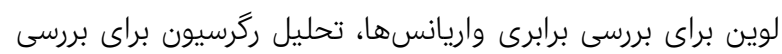

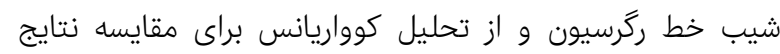
يس آزمون بين دو گروه در قالب نرمافزار آمارى SPSS 23 استفاده

\section{يافتهها}

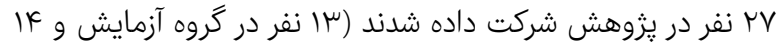

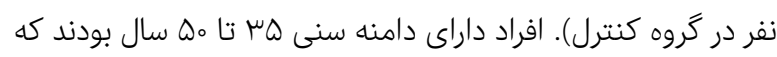

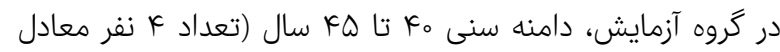

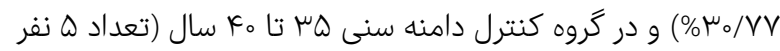

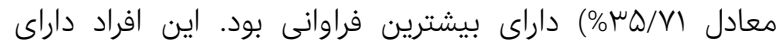

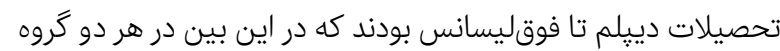

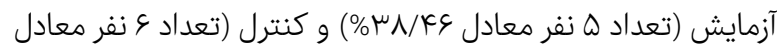

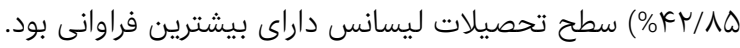

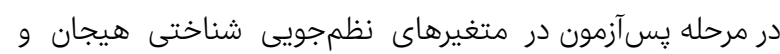

دادههاى يزوهش با استفاده از برسشنامهاههاى زير كَردآورى شدند: يرسشنامه نظمجويى شناختى هيجان (CERQ): توسط كروس و و

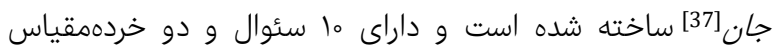

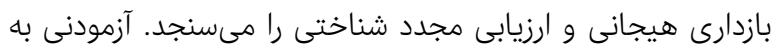

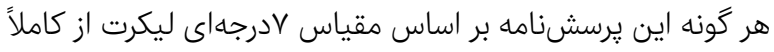

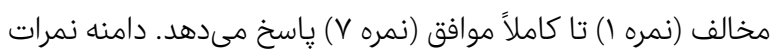

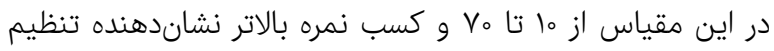

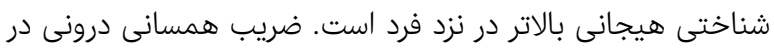

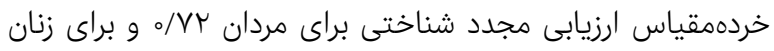

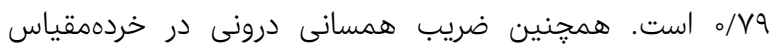

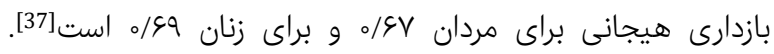

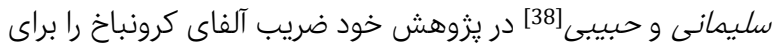

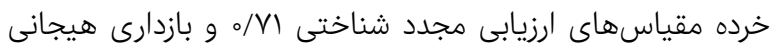

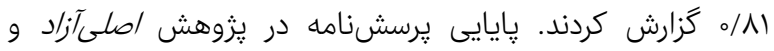

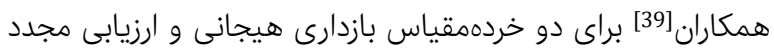

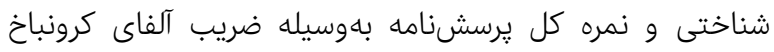

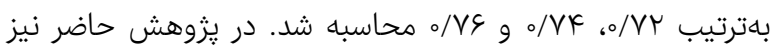

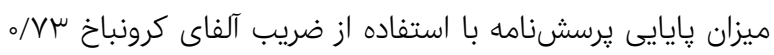
محاسبا شد. مقياس تابآورى (RS): توسط كانر و ديوديسون [40] براى سنجش

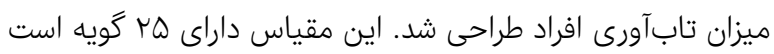

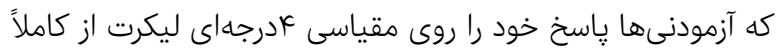

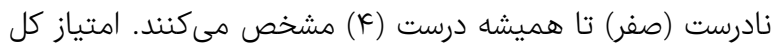

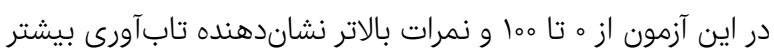

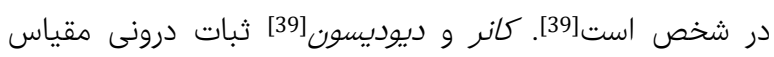

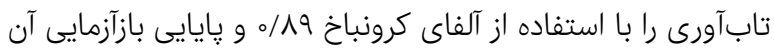

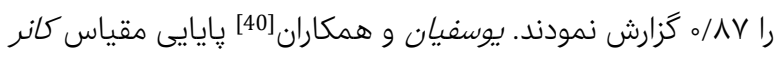

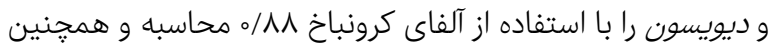

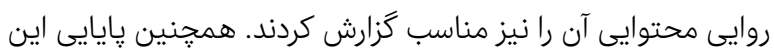

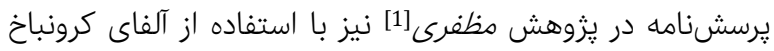

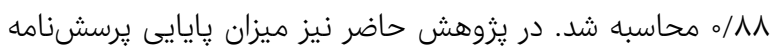

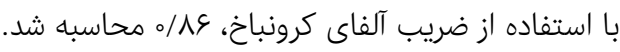

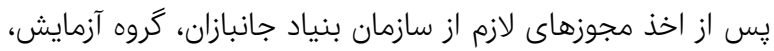

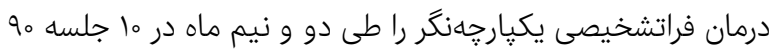

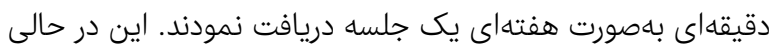

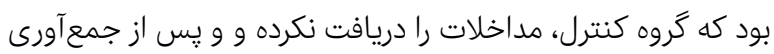

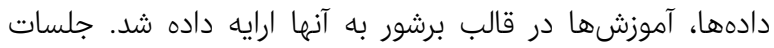

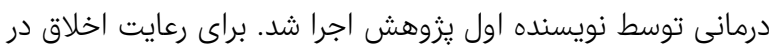

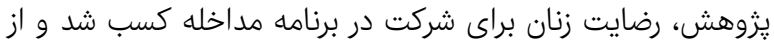

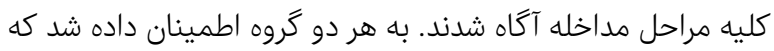

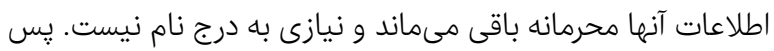

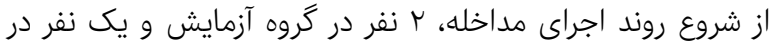
كروه كنترل از مطالعه خارج شدند. 
همكاران[27] مبنى بر اثربخشى درمان فراتشخيصى فراني بر ابعاد تنظيم

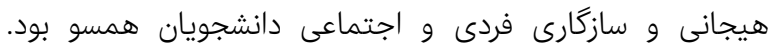

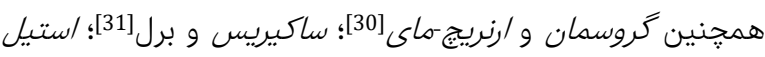

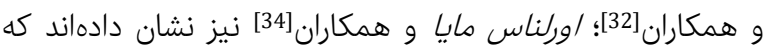

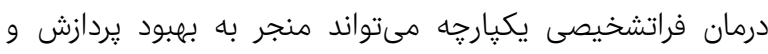

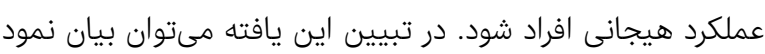

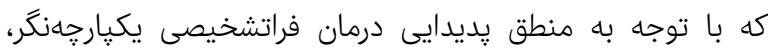

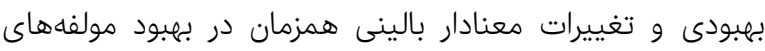
روانشناختى، هيجانى و ارتباطى مىتواند بهواسطه آماج قرارگرفتن

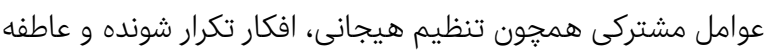
منفى باشد كه در مدلهاى يروتكل روى آنها تاكيد مى هُشود [31].

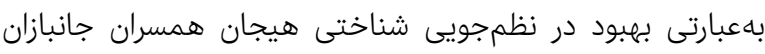

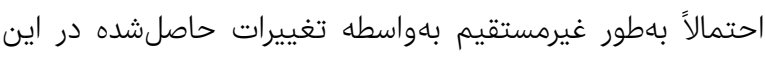

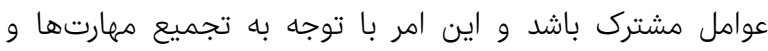
تكنيكهاى اثربخش بر اين عوامل مشترك يا فراتشخيصى برى براى إن

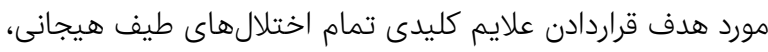

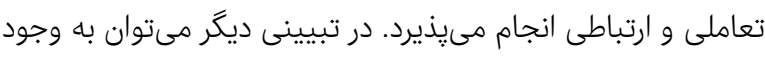

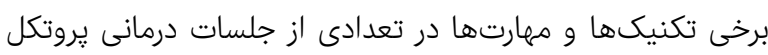

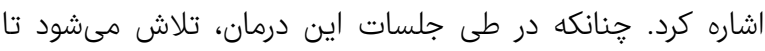

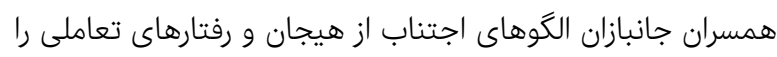

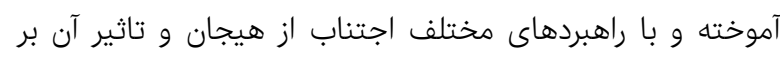

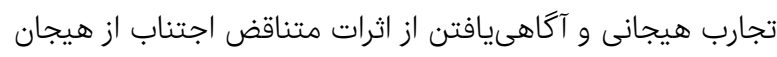

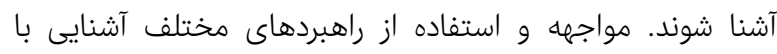
تجارب هيجانى سبب مىشود تا فرد با مهارت تنظيم هيجانى بالاتر،

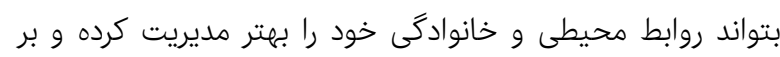

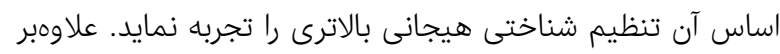

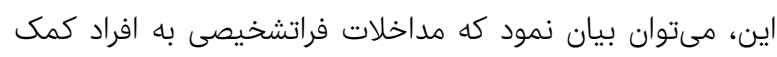

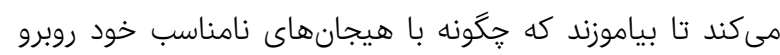

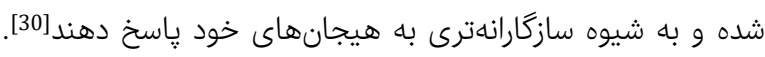

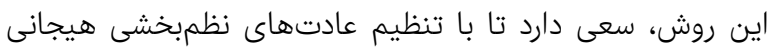

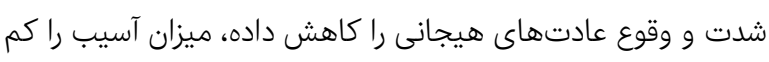

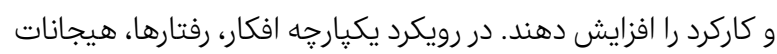

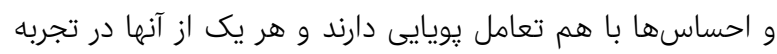

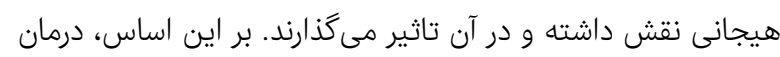

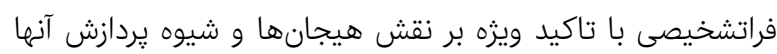

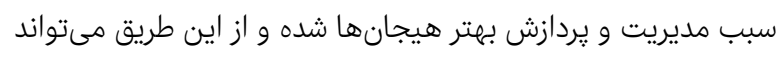
منجر به افزايش تنظيم شناختى هيجان در همسران جانبازان شود.

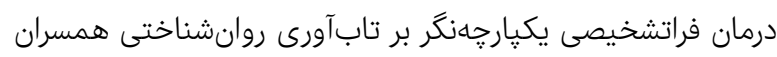

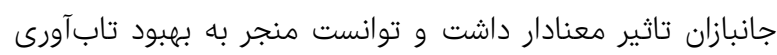

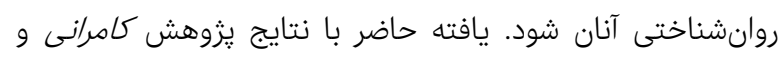

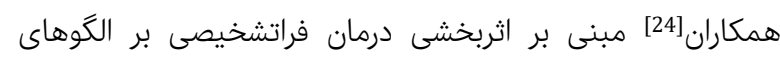

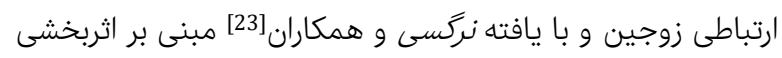

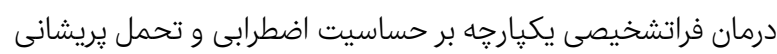

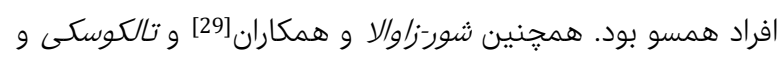

تابآورى روانشناختى يِيشفرض همگنى واريانسها برقرار بود

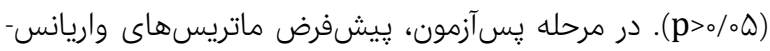

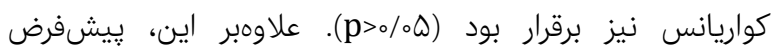

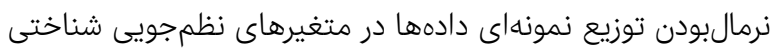

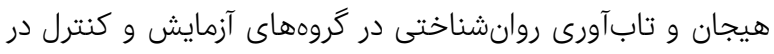

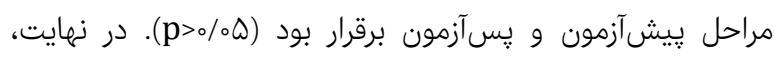

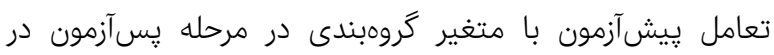

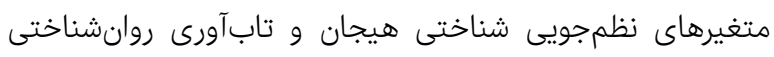

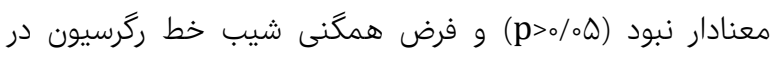

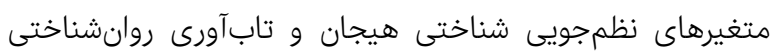

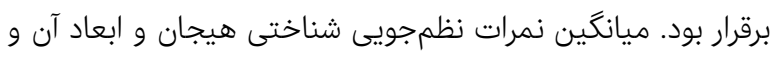

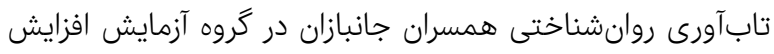

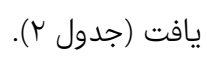

جدول r) مقايسه ميانكين نظمجويى شناختى هيجان و ابعاد آن و تابآورى دورى

\begin{tabular}{|c|c|c|c|}
\hline يسآزمون & ي ي يشآزمون & 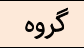 & مولفهها و ابعاد \\
\hline$F \Delta / \mu_{\circ} \pm F / Q r$ & $\mu V / \circ \circ \pm \Delta / \mathcal{A} I$ & آزمايش & \multirow{2}{*}{ نظمجويى شناختى هيجان } \\
\hline$\mu \varepsilon / q \mu_{ \pm} F / \mu I$ & $\mu V / T I \pm \Delta / \Delta V$ & كنترل & \\
\hline$r \varepsilon / 9 r \pm \mu / \mu \omega$ & $r Y / V V \pm K / O r$ & 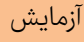 & \multirow{2}{*}{ بازدارى هيجانى } \\
\hline 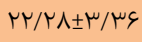 & $r K / G K \pm F / F K$ & كنترل & \\
\hline $\mid \Lambda / \mu \Lambda \pm \mu / \mu q$ & $\mid K / r \mu \pm r / \Lambda F$ & 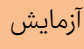 & \multirow{2}{*}{ رزيابى مجدد شناختى } \\
\hline $\mid K / \varepsilon F \pm r / k r$ & $\mid K / Q V \pm r / r K$ & كنترل & \\
\hline$\Delta Q / \Delta \mu \pm V / . \varphi$ & $K Q / \circ \pm \pm \lambda / V K$ & 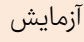 & \multirow{2}{*}{ نابآورى روانشناختى } \\
\hline$\kappa \kappa / ৭ r \pm \Delta / \wedge Q$ & $K \Delta / / F \pm S / 19$ & كنترل & \\
\hline
\end{tabular}

\% تVV

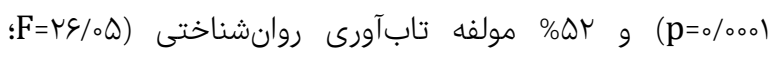

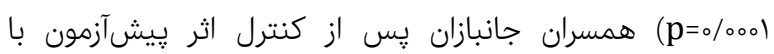

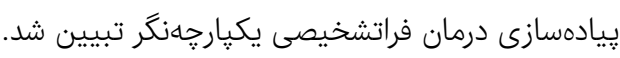

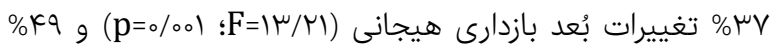

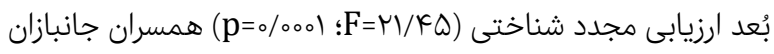

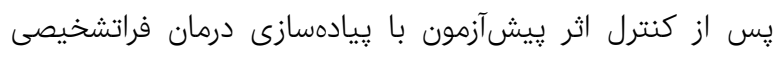

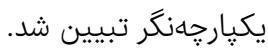

بحث مساله اصلى يزوهش حاضر بررسى اين نكته بود كه آيا بين درمان فراتشخيصى يكيارجهنگ

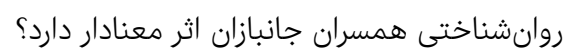

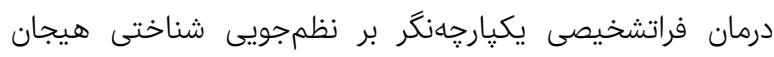

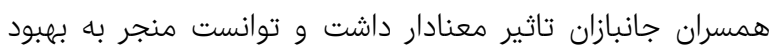

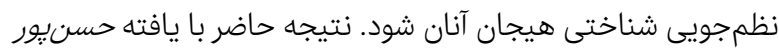

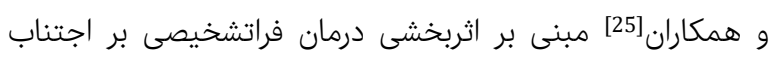

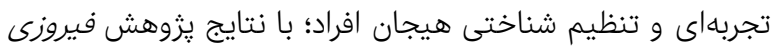

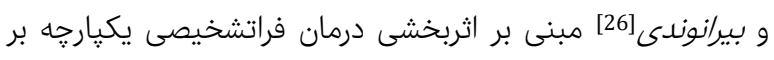

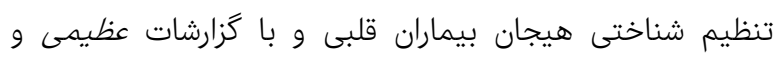


مىتواند باعنوان يك درمان كارآمد براى بهبود نظمجويى شناختى

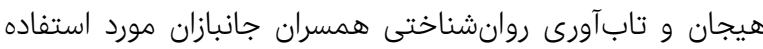
قرار گيرد. - مئ

تشكر و قدردانى: بدين وسيله از تمام زنانى كه در يزوهش شركت كردند

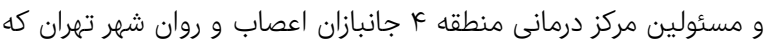

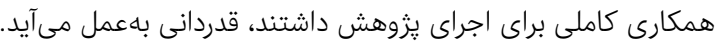
تاييديه اخلاقى: بيان نشد.

تعارض منافع: مقاله حاضر بركرفته از رساله دوره دكتراى تخصصى واليه تئليه

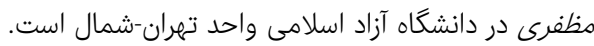

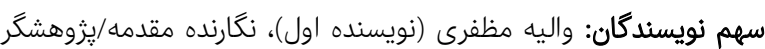

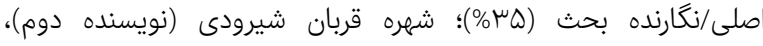

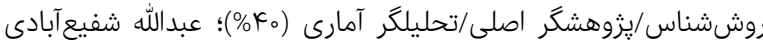

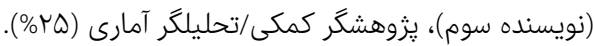

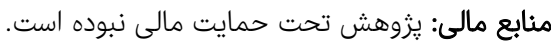

منابع

1- Mozaffari V. Effectiveness of cognitive mindfulnessbased-therapy on resilience and emotional control of psychiatric veteran's wives. Iran J War Public Health. 2019;11(2):61-6. [Persian]

2- Dehghan F, Piri Kamrani M, Goli R, Rahmani H. The impact of life skills training on marital satisfaction, marital dissatisfaction and mental health of veteran's wife. Q J Woman Soc. 2017;7(4):1-11. [Persian]

3- Showani E, Zahrakar K, Rasooli M. Effectiveness of acceptance and commitment therapy on reducing the components of couple burnout in veterans spouses. Iran J War Public Health. 2016;8(2):111-8. [Persian]

4- Abbasi S, Dokaneifard F, Shafiabadi A. The comparison of the effectiveness of emotionally focused therapy and cognitive behavioral therapy on family cohesion and marital satisfaction of veteran' wives in Khoramabad city. J Psychol Sci. 2019;17(71):823-9. [Persian]

5- Moghanloo M, Hosseini SM. Prediction of emotional empathy in the wives of veterans by cognitive emotion regulation strategies and resilience. Iran J War Public Health. 2019;11(2):93-9. [Persian]

6- Joormann J, Stanton CH. Examining emotion regulation in depression: A review and future directions. Behav Res Ther. 2016;86:35-49.

7- $\mathrm{Li} \mathrm{D}, \mathrm{Li} \mathrm{D}, \mathrm{Wu} \mathrm{N}$, Wang $\mathrm{Z}$. Intergenerational transmission of emotion regulation through parents' reactions to children's negative emotions: Tests of unique, actor, partner, and mediating effects. Child Youth Serv Rev. 2019;101:113-22.

8- Malesza M. Stress and delay discounting: The mediating role of difficulties in emotion regulation. Person Individ Differ. 2019;144:56-60.

9- Panayiotou G, Panteli M, Leonidou C. Coping with the invisible enemy: The role of emotion regulation and awareness in quality of life during the COVID-19 pandemic. J Context Behav Sci. 2021;19:17-27.

10- Herwig U, Opialla S, Cattapan K, Wetter TC, Jäncke L, Brühl $\mathrm{AB}$. Emotion introspection and regulation in depression. Psychiatry Res Neuroimaging. 2018;277:713.

11- Kirwan M, Pickett SM, Jarrett NL. Emotion regulation as a moderator between anxiety symptoms and insomnia symptom severity. Psychiatry Res. 2017;254:40-7.
همكاران[33] نشان دادهاند كه درمان فراتشخيصى يكيارجه منجر به بهبود قدرت روانى و مقابلهاى افراد مىشود. در تبيين اين يافته

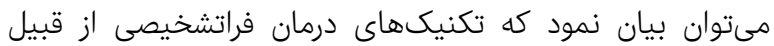

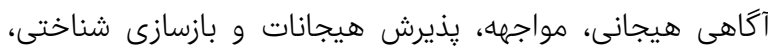

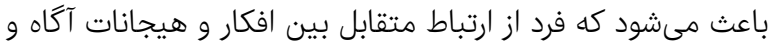

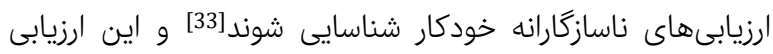
مجدد شناختى، باعث افزايش انعطافيذيرى در افكار و تعديل

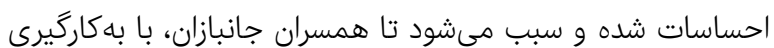
انعطافيذيرى شناختى، قدرت حل مساله خود را نيز بهبود بخشيدها

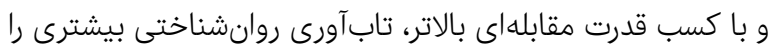
از خود نشان دهند. علاوهبر اين، مىتوان بيان نمود كه درمان بان بان

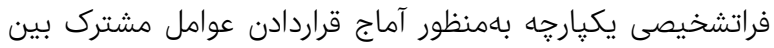

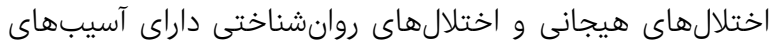

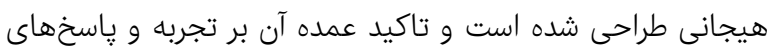

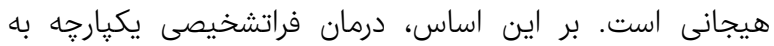
همسران جانبازان مىآموزد كه جگونه با هيجانات ناخوشايند خود

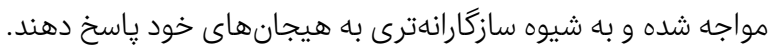
اين درمان كمك مىكند تا همسران جانبازان، فهم بهترى از تعامل افكار، احساسها و رفتارها در ايجاد تجربههاى هيجانى درونى هانى

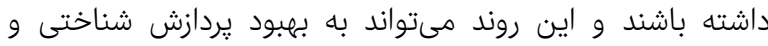

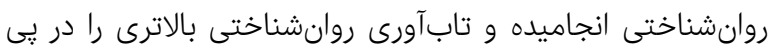

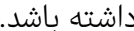

محدودبودن دامنه تحقيق به همسران جانبازان شهر تهران؛ عدم

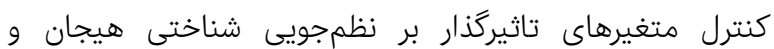

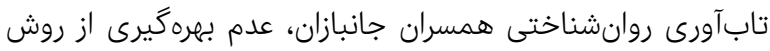
نمونه

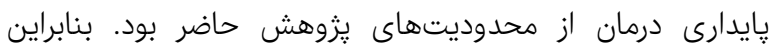

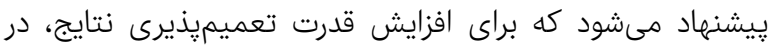

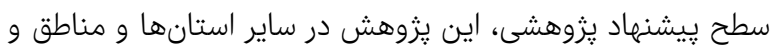

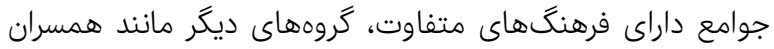

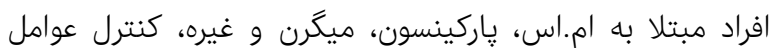

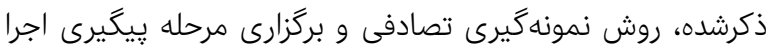

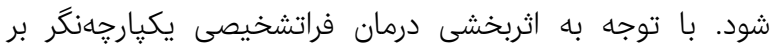

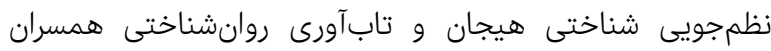

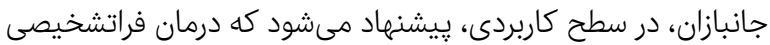

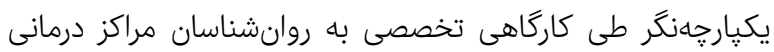

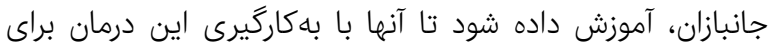

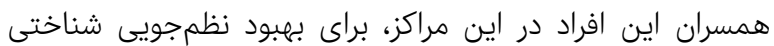
هيجان و تابآورى روانشناختى آنان گَامى عملى برداشته باشند. بران.

\section{نتيجه كيرى}

درمان فراتشخيصى يكيارجه با بهرهگيرى از فنونى همانند شناسايى

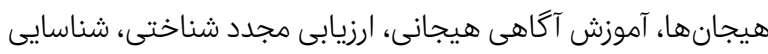

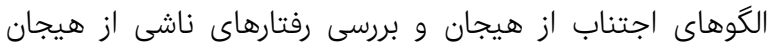


اثربخشى درمان فراتشخيصى يكيارجهنكر بر نظمجويى شناختى هيجان و ... 1ه

cardiovascular disease. YAFTE. 2018;20(2):93-102. [Persian]

27- Azimi A, Omidi A, Shafiei E, Nademi A. The effectiveness of transdiagnostic, emotion- focused treatment for emotional regulation and individual-social adjustment in female students. J Arak Uni Med Sci. 2018;20(10):62-73. [Persian]

28- Otared N, Mikaeili N, Mohajeri N, Vojoudi B. The effect of unified transdiagnostic treatment on anxiety Disord and comorbid depression: Single-case design. J Torbatheidarie Univ Med Sci. 2017;4(4):54-62. [Persian] 29- Sauer-Zavala S, Bentley KH, Steele SJ, Tirpak JW, Ametaj AA, Nauphal M, et al. Treating depressive disorders with the Unified Protocol: A preliminary randomized evaluation. J Affect Disord. 2020;264:438-45. 30- Grossman RA, Ehrenreich-May J. Using the unified protocol for transdiagnostic treatment of emotional disorders with youth exhibiting anger and irritability. Cogn Behav Pract. 2019;27(2):184-201.

31- Sakiris N, Berle D. A systematic review and metaanalysis of the Unified Protocol as a transdiagnostic emotion regulation based intervention. Clin Psychol Rev. 2019;72:101751.

32- Steele SJ, Farchione TJ, Cassiello-Robbins C, Ametaj A, Sbi S, Sauer-Zavala S, et al. Efficacy of the unified protocol for transdiagnostic treatment of comorbid psychopathology accompanying emotional disorders compared to treatments targeting single disorders. J Psychiatr Res. 2018;104:211-6.

33- Talkovsky AM, Green KL, Osegueda A, Norton PJ. Secondary depression in transdiagnostic group cognitive behavioral therapy among individuals diagnosed with anxiety disorders. J Anxiety Disord. 2017;46:56-64.

34- de Ornelas Maia ACC, Nardi AE, Cardoso A. The utilization of unified protocols in behavioral cognitive therapy in transdiagnostic group subjects: A clinical trial. J Affect Disord. 2015;172:179-83.

35- Liu X, Cao J, Xie C. Finite-time and fixed-time bipartite consensus of multi-agent systems under a unified discontinuous control protocol. J Franklin Instit. 2019;356(2):734-51.

36- Kline G, Honaker J, Joseph A, Scheve K. Analyzing Incomplete Political Science Data: An Alternative Algorithm for Multiple Imputation. American Political Science Review. 2001;95(1):49-69.

37- Gross JJ, John OP. Individual differences in two emotion regulation processes: Implications for affect, relationships, and well-being. J Person Soc Psychol. 2003;85(2):348-62.

38- Soleimani A, Habibi Y. Relationship between emotion regulation and resilience with psychological well-being in students. J Sch Psychol Q. 2015;3(4):51-72. [Persian]

39- Asli Azad M, Manshei G, Qamrani A. Effectiveness of acceptance and commitment therapy on cognitive emotion regulation and tolerance of uncertainty of the students with obsessive-compulsive disorder. Psychol Except Individ. 2020;9(36):33-53. [Persian]

40- Conner KM, Davidson JRT. Development of a new resilience scale: The conner-davidson resilience scale (CD-RISC). Depress Anxiety. 2003;18(2):76-82.

41- Yousefian Z, Ahadi H, Keraskian Mojmanari A. The effectiveness of spirituality therapy on psychological distress and resilience of thalassemia patients. J Psychol Sci. 2020;18(84):23-35. [Persian]
12- Moghtader L. Relationship between religious beliefs and stress coping strategies in psychological resilience and marital satisfaction of veterans wives. J Guilan Univ Med Sci. 2017;26(103):37-45. [Persian]

13- Diener E, Lucas R, Schimmack U, Helliwell J. Wellbeing for Public policy. Oxford: Oxford University Press; 2009.

14- Thomas LJ, Asselin M. Promoting resilience among nursing students in clinical education. Nurs Educ Pract. 2018;28:231-4.

15- Tlapek SM, Auslander W, Edmond T, Gerke D, Schrag $\mathrm{RV}$, Threlfall J. The moderating role of resiliency on the negative effects of childhood abuse for adolescent girls involved in child welfare. Child Youth Serv Rev. 2017;73:437-44.

16- Wright M, Masten AS, Narayan AJ. Resilience processes in development: Four waves of research on positive adaptation in the context of adversity. In: Goldstein S, Brooks RB, editors. Handbook of Resilience in Children. New York: Springer; 2013.

17- Steensma H, Heijer MD, Stallen V. Effects of resilience training on the reduction of stress and depression among Dutch workers. Int $\mathrm{Q}$ Commun Health Educ. 2007;27(2):145-59.

18- Dey NEY, Amponsah B. Sources of perceived social support on resilience amongst parents raising children with special needs in Ghana. Heliyon. 2020;6(11):e05569. 19- Backmann J, Weiss M, Schippers MC, Hoegl M. Personality factors, student resiliency, and the moderating role of achievement values in study progress. Learn Individ Differ. 2019;72:39-48.

20- Allen LB, McHugh RK, Barlow DH. Emotional disorders: A unified protocol. In: Barlow DH, Editor. Clinical handbook of psychological disorders: A stepby-step treatment manual. New York: Guilford Press; 2007.

21- Farchione TJ, Fairholme CP, Ellard KK, Boisseau CL, Thompson-Hollands J, Carl JR, et al. Unified protocol for transdiagnostic treatment of emotional disorders: A randomized controlled trial. Behavior Therapy. 2020;43(3):666-78.

22- Cassiello-Robbins C, Southward MW, Tirpak JW, Sauer-Zavala S. A systematic review of Unified Protocol applications with adult populations: Facilitating widespread dissemination via adaptability. Clin Psychol Rev. 2020;78:101852.

23- Nargesi F, Fathi Ashtiani A, Davoudi I, Ashrafi E. The effect of unfiled transdiagnostic treatment on anxiety sensitivity, distress tolerance and obsessive-compulsive symptoms in individuals with obsessive-compulsive disorder. J Psychol Achiev. 2019;28(2):49-66. [Persian]

24- Kamrani Z, Bahrami F, Goodarzi K, Farrokhi N. Effectiveness of transdiagnostic educational package on communication patterns and secure bond of couples referred to family counseling centers. J Psychol Sci. 2019;18(81):1057-67. [Persian]

25- Hasanpoor P, Aghausefi A, Zamir 0, Alipour A. The effectiveness of transdiagnostic treatment on experiential avoidance and cognitive emotion regulation in patients with obsessive-compulsive disorder and its comparison with exposure and prevention response therapy. J Clin Psychol. 2019;11(3):25-38. [Persian]

26- Firoozi M, Biranvandi M. Integrate metacognition for cognitive emotional regulation in patients with 